\title{
TDAH em adultos e suas implicações em âmbito acadêmico
}

\section{ADHD in adults and its academic implications}

\section{Michely Aparecida da Silva ${ }^{1^{*}}$, Tamires Jordão Laport ${ }^{2}$ \\ Como citar esse artigo. da SILVA, M. A.; LAPORT, T. J. TDAH em adultos e \\ Resumo} suas implicações em âmbito acadêmico. Mosaico - Revista Multidisciplinar de Humanidades, Vassouras, v. 12, n. 2, p. 34-40, mai./ago. 2021.

Nota da Editora. Os artigos publicados na Revista Mosaico são de responsabilidade de seus autores. As informações neles contidas, bem como as opiniões emitidas, não representam pontos de vista da Universidade de Vassouras ou de suas Revistas.

O Transtorno de Déficit de Atenção e Hiperatividade (TDAH) é um transtorno do neurodesenvolvimento que surge normalmente na infância, se estendendo até a vida adulta, podendo afetar questões de origem socioafetiva, acadêmica e profissional, além de ser um dos principais focos de estudo atualmente, tanto na área da saúde como da educação. Diante disso, o objetivo deste trabalho é discutir sobre a atuação do docente em âmbito acadêmico diante de alunos que apresentem características peculiares ao TDAH. O que se busca aqui é analisar a invisibilidade marcante na educação referente ao TDAH de jovens e adultos. Já que, atualmente, situações de alunos com dificuldades de aprendizagem e baixo rendimento acadêmico têm sido identificados com maior frequência. Além disso, o diagnóstico do TDAH é mais recorrente na infância, o que faz com que seu reconhecimento entre jovens e adultos não seja muito discutido. Trata-se, portanto, de uma revisão de literatura, tendo como fontes: artigos científicos, livros, trabalhos acadêmicos e periódicos on-line, com o intuito de investigar propostas de diferentes autores e com aproximação ao tema abordado, para a complementação de ideias e concepções.

Palavras-chave: TDAH; Transtornos mentais; Ensino Superior; Educação.

\begin{abstract}
Attention Deficit Hyperactivity Disorder (ADHD) is a neurodevelopmental disorder that usually arises in childhood, extending into adulthood, and may affect socio-affective, academic and professional issues, in addition to being one of the main focuses of study today, both in the area of health and education. Therefore, the aim of this paper is to discuss the role of the teacher in the academic field in the face of students who have characteristics peculiar to ADHD. What is sought here is to analyze the remarkable invisibility in the education related to ADHD of young people and adults. Since, currently, situations of students with learning difficulties and low academic performance have been identified more frequently. In addition, the diagnosis of ADHD is more recurrent in childhood, which means that its recognition among young people and adults is not much discussed. It is, therefore, a literature review, having as sources, scientific articles, books, academic works and online journals, in order to investigate proposals from different authors and with approximation to the topic addressed, to complement ideas and conceptions.
\end{abstract}

Keywords: ADHD; Mental disorders; University education; Education.

\section{Introdução}

De acordo com o Manual Diagnóstico e Estatístico de Desordens Mentais (APA, 2014), o TDAH caracteriza um estado neurobiológico refletido em níveis de desenvolvimento não apropriados de atenção, como condutas de hiperatividade e impulsividade. O TDAH engloba um panorama multifacetado, decorrência tanto do aparato biológico do sujeito, quanto da interação desse sujeito com o meio.

Atualmente, o TDAH é considerado um dos transtornos mentais mais estudados, tanto na área da educação, quanto da saúde. O TDAH é identificado como uma desordem neurobiológica que normalmente é diagnosticado na infância, acompanhando o sujeito por toda a vida. O indivíduo que apresenta TDAH, na maioria das vezes está envolto em uma tríade de sintomas: inquietude, impulsividade e déficit de atenção.

Aponta-se, que o cenário que mais reconhece sintomas de TDAH nos indivíduos é o cenário escolar. $\mathrm{O}$ aluno que é identificado com os sintomas recorrentes ao TDAH, normalmente tem um baixo rendimento escolar, dificuldade de aprendizagem, hiperatividade e falta de atenção.

Portanto, é imprescindível a valorização de um diagnóstico preciso, para que o aluno seja encaminhado para um tratamento adequado e que obtenha ajuda em seu processo de aprendizagem, com o intuito de contribuir com a sua inclusão escolar. (GONÇALVES, 2019)

O trabalho em questão abordará a atuação do docente de nível superior, no convívio com alunos que apresentem características peculiares ao TDAH,

Afiliação dos autores

${ }^{1}$ Discente do curso de Psicologia, Universidade de Vassouras, Rio de Janeiro, Brasil

${ }^{2}$ Psicóloga, mestre em Psicologia pela Universidade Federal de Juiz de Fora, Minas Gerais, Brasil, docent4e do curso de Psicologia da Universidade de Vassouras, Rio de Janeiro, Brasil. 
identificando comportamentos dos professores no reconhecimento dos sintomas de TDAH nesses alunos e nas possibilidades (ou não) de suporte. $\mathrm{O}$ que se busca aqui é discutir sobre essa invisibilidade marcante na educação referente ao TDAH de jovens e adultos.

Sendo assim, o trabalho será movido a partir da seguinte questão norteadora: Como o aluno com TDAH pode ser tratado no círculo acadêmico pelos professores? Se, por vezes, no ensino básico os professores tratam de outra forma.

Trata-se de um trabalho de cunho bibliográfico, tendo como base metodológica, artigos, livros, trabalhos acadêmicos e periódicos on-line, a fim de coletar propostas de diversos autores que se aproximem do tema em questão, avaliando suas ideias e concepções.

Percebe-se, no entanto, que na literatura pesquisada, a quantidade de material encontrada referente ao tema é escassa. Sendo este um dos motivos que justificaram a escolha do tema. Enquanto o TDAH em adultos no Ensino Superior é um assunto pouco discutido na seara acadêmica, outros temas e/ou contextos que envolvam o TDAH são frequentemente abordados.

Nessa perspectiva, o artigo em questão teve como objetivo geral analisar a atuação do docente no Ensino Superior diante de alunos que apresentem características peculiares ao TDAH. E, em contrapartida, como objetivos específicos, obtendo mais informações a respeito do TDAH; analisando a invisibilidade marcante na educação referente ao TDAH de jovens e adultos; e, por fim, apresentando aspectos que justifiquem essa diferenciação de tratamento nos diferentes ensinos.

\section{TDAH: Conceito e especificidades}

Aponta-se, que a desordem de déficit de atenção foi nomeada de diversas formas com o passar do tempo. A terminologia Transtorno de Déficit de Atenção/ Hiperatividade (TDAH), como foi sugerida pelo Manual Diagnóstico e Estatístico de Desordens Mentais (DSM-IV-TR) da American Psychiatric Association APA (APA, 2014) manifesta um estado neurobiológico, que diz respeito aos níveis de desenvolvimento não apropriados de atenção, comportamento hiperativo e impulsividade. O TDAH apresenta uma condição multifacetada, decorrente de dois fatores: biológico e da interação do indivíduo com o meio em que vive. (FERREIRA, 2011)

De acordo com o DSM-V-TR (APA, 2014), existem três subtipos de TDAH. O primeiro subtipo, que é o subtipo combinado, é o mais conhecido e o mais recorrente também, que caracteriza o comportamento hiperativo e impulsivo, associado à desatenção. $\mathrm{O}$ segundo subtipo é marcado pela falta de atenção, caracterizando-se em cometer uma grande repetição de erros por conta da falta de atenção aos detalhes ou perder total atenção facilmente, o que acaba sendo um comportamento negligenciado, considerado como um quadro de devaneio e desorganização. E, por último, o terceiro subtipo, que tem a hiperatividade como comportamento predominante, a agitação e a impulsividade, bem parecido com o primeiro subtipo. Os indivíduos desse subtipo são identificados por sua inquietude, procuram realizar várias atividades ao mesmo tempo, apresentam comportamentos explosivos e não sabem lidar com frustrações. (FERREIRA, 2011) Como Silva (2010) aponta:

O TDAH se caracteriza por três sintomas básicos: desatenção, impulsividade e hiperatividade física e mental. Costuma se manifestar ainda na infância e em cerca de $70 \%$ dos casos o transtorno continua na vida adulta. Ele acomete ambos os sexos, independentemente do grau de escolaridade, situação socioeconômica ou nível cultural, o que pode resultar em sérios prejuízos na qualidade de vida das pessoas que o têm, caso não sejam diagnosticadas e orientadas precocemente. (SILVA, 2010, p. 8)

Normalmente, as crianças que possuem características específicas ao TDAH, podem ser introspectivaseapresentarcomplicaçõesdememorização (GONÇALVES, 2019). Portanto, é possível afirmar, que essas crianças têm a capacidade de aprender, mas as dificuldades em relação à produtividade e o rendimento escolar são maiores, consequência da influência que os sintomas do TDAH provocam. (FERREIRA, 2011)

Cabe ressaltar, que o TDAH costuma se manifestar na infância, especialmente ao iniciar a vida escolar, onde os sintomas se revelam de forma mais nítida, normalmente percebendo as dificuldades na aprendizagem do padrão de desenvolvimento esperado do aluno para a sua idade. (LOPES, 2011)

Aponta-se, que o Distúrbio de Déficit de Atenção (DDA) é um distúrbio biopsicossocial, que diz respeito a grandes aspectos genéticos, biológicos e sociais (TOPCZEWSKI, 2012 apud GONÇALVES, 201). Portanto, o TDAH é caracterizado por uma tríade, composta pela falta de atenção, hiperatividade e impulsividade. (GONÇALVES, 2019)

Salienta-se, que o TDAH não é resultante de uma única causa identificável, já que as evidências científicas ao longo dos anos apontam para diversos fatores em relação aos seus sintomas. (MATTOS, 2007 apud GONÇALVES, 2019)

Como Gonçalves (2019) menciona:

O Transtorno de Déficit de Atenção e Hiperatividade (TDAH) é um transtorno no desenvolvimento do autocontrole que consiste em problemas em relação aos períodos de atenção, ao controle do impulso e ao nível de atividade, acometendo cerca de 3 a $6 \%$ das crianças desde tenra idade e persistindo na vida adulta em mais da metade dos casos. De acordo com o DSM-V (Manual de Diagnóstico e Estatístico de 
Transtornos Mentais), o TDAH classifica-se entre os transtornos do neurodesenvolvimento caracterizados por dificuldades no desenvolvimento e se manifestam de forma precoce, influenciando o comportamento pessoal, social ou acadêmico. (GONÇALVES, 2019, p. 21)

Para entender melhor esse quadro, é importante dissociar os sintomas do TDAH das definições equivocadas que dizem respeito à dificuldade de aprendizagem e baixa produtividade escolar. $\mathrm{O}$ que deve ser levado em conta, é que a dificuldade em realizar uma tarefa entende-se como falta de capacidade para compreender conteúdos específicos. Em relação ao TDAH, a falta de atenção sobre o conteúdo escolar pode acarretar desempenho insuficiente para as tarefas estipuladas, mas, mesmo assim a capacidade para a realização dessas funções é presente. (FERREIRA, 2011)

Segundo Lopes (2011), as crianças que apresentam relação com o TDAH, possuem atraso psicomotor e grande dificuldade em seguir instruções e regras, além de não conseguirem se manter concentrados em tarefas pouco motivadoras.

Em resumo, de acordo com Gonçalves (2019):

As características mais comuns do TDAH são: a dificuldade em se manter o foco em alguma atividade que exija esforço mental prolongado ou em alguma tarefa que precise ser realizada com regras e prazos pré-determinados. Outra dificuldade apresentada diz respeito a começar e terminar as tarefas escolares. Crianças com TDAH são desatentas, esquecidas e desorganizadas, o que pode levar a um baixo rendimento escolar. (GONÇALVES, 2019, p. 22)

Em se tratando do diagnóstico do TDAH, é necessário que se tenha grande cautela e experiência, já que somente com um diagnóstico preciso é possível obter um tratamento efetivo. Nesse contexto, o diagnóstico da criança é feito diante de dois aspectos: as informações obtidas na avaliação médica e os dados da história da criança. A partir disso, fatores estarão associados a dois sinais e sintomas: falta de atenção e a hiperatividade/ impulsividade. Portanto, é essencial que se observe com cautela a distinção entre a falta de atenção primária e secundária, associada às condições de vida de cada criança. (GONÇALVES, 2019)

O diagnóstico, portanto, é realizado através da anamnese, que diz respeito a uma conversa composta por mais detalhes, entre o médico e os responsáveis pela criança, sobre a história de vida dessa criança, desde sua gestação até os dias atuais. (SILVA, 2014 apud GONÇALVES, 2019)

Portanto, é fundamental que o diagnóstico seja coerente, para que se estabeleça o quanto antes, diante dos sintomas apresentados, a diferença entre o transtorno, de fato, ou apenas uma fase pela qual a criança está passando (GONÇALVES, 2019). Quanto mais cedo o diagnóstico for concluído, melhor, pois dificuldades maiores em sua vida escolar e sua interação social poderam ser evitadas e/ou amenizadas. Por isso a grande relevância do acompanhamento da família, dos profissionais de educação e de outros profissionais conhecerem o TDAH.

A partir de então, um plano de tratamento é estipulado, com base nos dados adquiridos na avaliação. Assim, o comportamento escolar e o desempenho da criança são avaliados, para então definir o êxito e a necessidade de mudanças no programa de intervenção. (LOPES, 2011).

\section{TDAH em adultos}

As crianças, desde a sua iniciação escolar são observadas com um olhar mais atento em relação à sua aprendizagem e comportamento dentro da escola, levando em conta também suas interações sociais. Sendo assim, torna-se mais fácil identificar crianças que possuam TDAH. No entanto, o transtorno também é presente em adultos, indivíduos que não foram diagnosticados na infância, ou que tenham TDAH desde a infância, mas não recebem a mesma atenção que uma pessoa que tenha sido diagnosticada com TDAH durante a infância.

É comum ouvirmos falar em crianças hiperativas, que são agitadas o tempo todo, que correm por todos os lados, sobem em móveis e que vivem "ligadas" o tempo inteiro, ou aquelas que são extremamente distraídas, desajeitas e que perdem a atenção ou se distraem por qualquer detalhe, por menor que seja. Que apresentam dificuldades de aprendizagem e interação social, tornando a sala de aula um campo de batalha, provocando a incompreensão de pais, amigos e professores. (SILVA, 2010)

No entanto, apesar de também ser comum adultos apresentarem sintomas bem parecidos, mas com certas particularidades, como desorganização, impaciência, hiperatividade, o fato de iniciarem diversos projetos ao mesmo tempo e abandonarem no meio do caminho, apresentarem altos e baixos repentinos, impulsividade, esquecerem com frequência de compromissos importantes, etc, nem sempre recebem a atenção devida a esses problemas como um indivíduo recebe na infância, acarretando certa invisibilidade de adultos que podem ter TDAH dependendo da intensidade e frequência dos comportamentos. (SILVA, 2010)

Como Schmitz et al. (2007) questionam:

Por que alguns casos de TDAH remitem enquanto outros persistem até a idade adulta? Há muito poucos dados na literatura que ajudam a esclarecer tal dúvida. Agrande maioria dos estudos que consideraram os preditores de persistência do TDAH teve como foco a persistência até a adolescência final. Gravidade do TDAH na infância, comorbidade com outros diagnósticos na infância e adolescência, aspectos de adversidade na infância, história familiar de TDAH e 
comorbidades psiquiátricas são preditores consistentes de persistência do transtorno. (SCHMITZ et al., 2007, p. 27)

Acreditava-se, que o TDAH perdurava apenas somente até a adolescência, no entanto, atualmente, já existem estudos científicos e comprovados, de que o TDAH persiste com grande frequência em adultos que foram diagnosticados com TDAH quando crianças (SCHMITZ et al., 2007). Percebe-se, no entanto, ainda certa deficiência em estudos na literatura sobre o padrão dessa ascensão dos sintomas e, em especial, sobre os aspectos que antecedem esse desfecho.

Aponta-se, que o TDAH na infância é um fator de risco de uma série de consequências na fase adulta, especialmente quando há comorbidades. Podendo se tornar um adulto com grandes problemas na vida pessoal e profissional, como maior número de divórcios, menor nível socioeconômico, mais mudanças de emprego, baixo desempenho acadêmico, dificuldades emocionais, etc. Mesmo assim, ainda não há uma concepção mais abrangente a respeito das variáveis que estejam relacionadas a essa permanência do TDAH até a vida adulta. (SCHMITZ et al., 2007)

De acordo com Lopes, Nascimento e Bandeira (2005), pesquisas apresentam, que em média, $67 \%$ de crianças com TDAH permanecem com os seus sintomas até a fase adulta, comprometendo a vida acadêmica, profissional, afetiva e social. Portanto, é essencial que a avaliação seja feita o quanto antes, para que o tratamento adequado seja oferecido e os sintomas reduzidos significativamente. (LOPES; NASCIMENTO; BANDEIRA; 2005) (2005):

De acordo com Lopes, Nascimento e Bandeira

Os estudos atuais têm identificado vários sintomas em adultos com diagnóstico de TDAH. Eles podem apresentar dificuldades com relações afetivas instáveis (separações, divórcios); instabilidade profissional que persiste ao longo da vida; rendimentos abaixo de suas reais capacidades no trabalho e na profissão; falta de capacidade para manter a atenção por um período longo; falta de organização (carente de disciplina); insuficiente capacidade para cumprir o que se comprometem; incapacidade para estabelecer cumprir uma rotina; esquecimentos, perdas e descuidos importantes; depressão e baixa autoestima; dificuldades para pensar e se expressar com clareza; tendência a atuar impulsivamente e interromper os outros; dificuldades de escutar e esperar sua vez de falar; frequentes acidentes automobilísticos devido à distração; frequente consumo de álcool e abuso de substância. (LOPES; NASCIMENTO; BANDEIRA; 2005, p. 67)

O TDAH em adultos muitas vezes tem sido visto como uma doença camuflada, por conta dos sintomas, que podem não estar aparecendo de forma clara, mas que acarretam em problemas de relacionamento afetivo e interpessoal, de organização, problemas de humor, abuso de substâncias, ou seja, caracterizados pela comorbidade. Com isso, o diagnóstico se torna mais complexo, e, em muitos casos, os adultos acabam ficando sem diagnóstico e, assim, sem tratamento. (LOPES; NASCIMENTO; BANDEIRA; 2005)

Sendo assim, é imprescindível que os comportamentos que possam indicar o transtorno em adultos, exijam uma atenção e estudos ainda maiores. Os sinais do TDAH englobam impulsividade e déficit de atenção, refletidos em certas dificuldades para dar curso e organização em um trabalho, se distraem facilmente, incapacidade de se concentrar, entre outros. Muitos adultos relacionam ainda, uma baixa autoestima associada ao baixo desempenho que afeta relações interpessoais e ocupacionais.

Além disso, ainda sofrem de transtorno depressivo secundário. Por isso, a terapia psicofarmacológica pode necessitar de cuidados frequentes, e deve ter acompanhamento em relação ao efeito da medicação e à aderência ao tratamento. A ideia é minimizar os sintomas da impulsividade e melhora no humor. (LOPES; NASCIMENTO; BANDEIRA; 2005)

De acordo com Schmitz et al. (2007) em seu estudo bibliográfico realizado acerca da remissão do TDAH na adolescência e preditores de persistência em adultos, pessoas com a junção de comportamentos (falta de atenção e hiperatividade/impulsividade) durante a infância, apresentaram maior índice de risco de persistência do TDAH em comparação àqueles com sintomas isolados de um grupo ou de outro, ou seja, dentro dos sintomas combinados, os indivíduos que apresentaram pelo menos seis sintomas de desatenção mais alguns sintomas de hiperatividade/impulsividade (subliminar) foram os que mostraram maiores chances de permanência do TDAH até a vida adulta.

Segundo Mattos (2003) citado por Lopes, Nascimento e Bandeira (2005)

Adultos com TDAH apresentam uma tendência pronunciada
de distração, esquecimento, repetições de erros, além
de perderem coisas, não recordarem o que acabaram de
ler, de necessitarem perguntar muitas vezes o mesmo e
evitarem sistematicamente toda leitura que não seja do seu
interesse específico. Geralmente envolvem-se em atividades
de pouca atenção e concentração por apresentarem tais
dificuldades. Isso não significa não prestar atenção nunca,
mas em muitas ocasiões, ou na maioria delas a pessoa
está dispersa, "no mundo da lua". (MATTOS, 2003, apud
LOPES; NASCIMENTO; BANDEIRA, 2005, p. 68)

Por tudo isso, infere-se, que embora o TDAH na infância e na adolescência sejam os mais investigados, ainda existem áreas com deficiência de informações mais abrangentes e consistentes em relação ao TDAH em adultos. Afinal, a ascensão nesse campo de estudos, acarretará em um melhor atendimento dos pacientes com TDAH, especialmente os adultos. (SCHMITZ et al., 2007). 


\section{Implicações do TDAH no Ensino Superior e sua invisibilidade na educação}

A princípio, é importante salientar, que há, em estudos científicos e pesquisas vigentes, certa deficiência em temas que abordem o TDAH dentro do Ensino Superior, discutindo sobre a relação de alunos que possuem o transtorno e seu desempenho acadêmico, aprendizagem e certos comportamentos.

Sendo assim, é possível perceber, a invisibilidade que adultos, portadores de TDAH sofrem dentro da Universidade, às vezes, sem receber tratamento adequado, como recebem crianças que também possuem o transtorno e em sua fase escolar.

Entende-se, portanto, que os adultos dentro do Ensino Superior, que venham a ter TDAH, por terem sido diagnosticados desde a infância, ou por precisarem de um olhar mais atento, necessitando do diagnóstico e tratamento nesse momento, não recebem essa análise mais detalhada por conta da invisibilidade que sofrem dentro da educação.

Como mencionado anteriormente, o diagnóstico de TDAH em adultos ainda é motivo de certos impasses, alguns estudos ainda mencionam o TDAH como transtorno exclusivo da infância e adolescência, sem persistir até a vida adulta, o que já foi diagnosticado e comprovado por estudos mais recentes. No entanto, mesmo que de maneira pouco incisiva, é reconhecido no texto do DSM-IV (APA, 2014) que em alguns casos, o TDAH possa permaneccer até a vida adulta. (MATTOS et al., 2006)

Como Mattos et al. (2006) apontam:

Estudos longitudinais demonstraram que o TDAH persiste na vida adulta em torno de 60 a $70 \%$ dos casos, sendo as diferenças encontradas nas taxas de remissão mais bem atribuídas às diferentes definições de TDAH ao longo do tempo do que ao curso do transtorno ao longo da vida e critérios diagnósticos mais restritos associados a taxas menores de persistência na vida adulta. [...] As alterações neurobiológicas em adultos com TDAH, incluindo os padrões de transmissão genética e os achados em estudos neuropsicológicos e de neuroimagem, são semelhantes àquelas encontradas em crianças e adolescentes com o transtorno, o que consolida a validade da forma adulta. (MATTOS et al., 2006, p. 51)

Em uma pesquisa de abordagem qualitativa e do tipo exploratória e de campo realizada por Lopes (2011), é apontado que $80 \%$ dos professores que participaram da pesquisa, admitiram que crianças com TDAH devam ter um tratamento especializado. O que também vale para alunos adultos, especialmente que estejam matriculados dentro de algum curso do Ensino Superior.

O professor deve ser uma das ferramentas primordiais dentro do ensino, buscando no aluno que tenha o transtorno, o interesse em alcançar conhecimentos, fazendo com que ele seja visto como qualquer outra pessoa e se sinta acolhido e seguro. Essa forma de conduzir o ensino e proceder diante de um aluno que tenha o transtorno, vai tornar o docente mais próximo e sensível. Assim, quando os alunos forem retirar suas dúvidas, estarão livre de constrangimentos e se interessam ainda mais pela aprendizagem (CARVALHO, 2000 apud LOPES, 2011). Portanto, a preocupação do professor em como trabalhar de forma especializada com alunos que tenham TDAH, deve levar em conta, antes de tudo, o reconhecimento da necessidade de atenção.

É imprescindível, que além da Universidade e dos docentes, a família também esteja engajada nesse processo de ensino e aprendizagem do aluno com TDAH. Afinal, o transtorno traz grandes influências na aprendizagem. Assim, uma atuação docente que não seja adequada, acarretará em grandes complicações não somente dentro do círculo acadêmico e seu processo de aprendizagem, como também na vida pessoal e profissional, afetando o convívio social e familiar. (LOPES, 2011)

A Associação Brasileira do Déficit de Atenção ABDA recomenda que a criança portadora de TDAH deva passar por um tratamento diferenciado em conjunto com a escola e a família, com o intuito em melhorar os níveis de qualidade de vida e aprimorar o desempenho escolar dessas crianças, buscando um grau maior de atenção a esses alunos/filhos. (LOPES, 2011). O que também torna viável a aplicação desse processo a acadêmicos do Ensino Superior portadores de TDAH.

Em se tratando do papel desempenhado pelo professor em sala de aula, algumas estratégias são atribuídas para que a aprendizagem dos alunos com TDAH seja potencializada, tais como: A valorização da participação desses alunos de maneira diferenciada; oferecer maior atenção; tratamento individualizado; reconhecimento das suas singularidades; tratá-lo de forma que não se sinta diferente dos outros; ser acolhedor e paciente; conhecer a capacidade do aluno; pesquisar sobre novos métodos que ajudem na sua aprendizagem; realizar atividade que prendam a sua atenção, entre outras. (LOPES, 2011)

No entanto, essa não é uma tarefa fácil, leva tempo, prática, e acima de tudo, atenção, é necessário que os docentes levem em conta, que os alunos, portadores de TDAH necessitam de maior atenção em seu processo de aprendizagem. Além disso, cada caso tem suas especificidades e grau de complexidade, e a maioria dos professores não é especializada em executar metodologias diante da inclusão no ensino.

Como é mencionado na pesquisa feita por Lopes (2011):

Viu-se que a grande maioria dos professores entrevistados tem conhecimento sobre as técnicas mais usuais e indicadas ao trabalho com pessoas com TDAH, em raros casos entre 
os pesquisados, foi verificado o total desconhecimento em relação a formas especificas de trabalho com essas crianças. É necessário lembrar sempre que crianças nessa situação passam por sérios problemas de convívio e de ajustamento social, cabendo ao professor ter conhecimento das técnicas que possibilitam a melhoria do processo de inclusão escolar. (SILVA, 2009 apud LOPES, 2011, p. 29)

No que diz respeito à avaliação do TDAH na escola, esta envolve diversas técnicas que compreendem a uma variedade de contextos e fontes de informações. A criança com sintomas associados ao TDAH é conduzida pelo professor para uma avaliação mais efetiva e relacionada a cinco etapas: (1) triagem de sintomas de TDAH; (2) avaliação multimodal; (3) interpretação dos resultados para chegar a decisão de classificação; (4) desenvolvimento de um plano de tratamento; e (5) avaliação contínua do programa de intervenção (LOPES, 2011). Cabe ressaltar, que o intuito da avaliação não é somente diagnosticar o TDAH, como também, elaborar um plano de intervenção com probabilidade em fornecer um tratamento efetivo de acordo com os dados coletados.

Diante de uma análise sobre a questão estrutural da educação, que envolve a formação e o preparo dos professores para a assitência de certas necessidades, tendo em vista o despreparo de alguns docentes em lidar com complicações presentes em sala de aula, favorece para a configuração do quadro. Normalmente, o plano educacional da escola também influencia, adotando um único tipo de enquadramento dos alunos no processo pedagógico. É percebido, que uma grande parcela dos professores aponta algum tipo de problema enfrentado diariamente no ambiente escolar em relação aos alunos com TDAH. (LOPES, 2011)

Portanto, é necessário que os professores reconheçam as necessidades dos alunos com TDAH, na busca em desenvolver metodologias de ensino que contribuam com a interação desses alunos com os seus colegas e com o próprio processo de ensinoaprendizagem. (LOPES, 2001 apud GOLDSTEIN, 2006)

É apontado ainda, que alunos com TDAH desenvolvem fatores de risco em uma diversidade de áreas funcionais. É como se os comportamentos dentro do transtorno, como desatenção, impulsividade e hiperatividade servissem de porta de entrada para outras dificuldades, que, em certos casos, apresentam uma gravidade maior do que os déficitis primordiais do TDAH. (DUPAUL; STONER, 2007 apud LOPES, 2011)

Sendo assim, é essencial que toda equipe de docentes esteja engajada em prol do auxílio e suporte aos outros integrantes da equipe multiprofissional que deve acompanhar os alunos com TDAH. Envolvendo, claro, a relação dos pais e responsáveis em um trabalho de parceria com a escola, oferecendo atenção e colaboração, pois, como Mattos et al. (2006) mencionam, em alguns casos, o transtorno persiste até a vida adulta, indicando que a minimização dos sintomas de hiperatividade impulsividade ao final da adolescência poderia ser parcial, sem remissão integral do TDAH, ao contrário do que se acreditava antes.

\section{Considerações finais}

Por tudo isso, infere-se que mesmo que a educação inclusiva tenha sido alvo de diversos progressos, contribuindo com um ensino longe de discriminações, adotando metodologias que se adequem à capacidade psíquica e funcional de alunos que necessitem de um tratamento adequado dentro do ensino regular, promovendo a equidade e a igualdade, valorizando a diversidade, ainda há grandes mudanças que precisam ser realizadas, principalmente dentro do Ensino Superior.

O TDAH é um transtorno geralmente reconhecido na infância, a criança ao iniciar sua vida escolar é observada com maior atenção e cuidado, e qualquer comportamento fora do padrão de desenvolvimento esperado do aluno para a sua idade é investigado. Os professores e os responsáveis acabam percebendo certas complicações educacionais e comportamentais dentro da rotina dessas crianças.

No entanto, o mesmo cuidado e preocupação não é visto em âmbito acadêmico universitário, os docentes não reconhecem nitidamente alunos que apresentem algum tipo de transtorno, como é o caso do TDAH. E mesmo que seja reconhecido, esses alunos não recebem um tratamento diferenciado.

O intuito aqui é reconhecer, como abordado por certos autores, que o TDAH persiste até a vida adulta em alguns casos, e, que, em se tratando do processo de ensino e aprendizagem e sua interação social, podem apresentar os mesmos sintomas que as crianças, como desatenção, impulsividade e hiperatividade, mas com certas especificidades, refletidos em sua vida pessoal e profissional.

A partir de então, é essencial que os professores e toda equipe multidisciplinar, ofereça todo o suporte e auxílio adequado aos alunos do Ensino Superior que sejam diagnosticados com TDAH, que levem em conta, a grande dificuldade que os sintomas do transtorno pode provocar nesses alunos.

\section{Referências}

AMERICAN PSYCHIATRIC ASSOCIATION. DSM-V. Manual diagnóstico e estatístico de transtornos mentais. American Psychiatric Association. Tradução: Maria Inês Corrêa Nascimento et al. Revisão técnica: Aristides Volpato Cordioli et al - 5. ed. - Dados eletrônicos. - Porto Alegre: Artmed, 2014.

FERREIRA, Patrícia Villa da Costa. Uma Revisão Teórica sobre o Transtorno de Déficit de Atenção e Hiperatividade (TDAH) e Estratégias Educacionais 
de Atendimento ao Aluno com TDAH. Revista de Psicologia, Fortaleza, v. 2 n. 2, p. 57-75, jul./dez. 2011. Disponível em: http://www.periodicos.ufc.br/ psicologiaufc/article/view/91. Acesso: 15 maio. 2021.

GONÇALVES, Kátia Cristiane dos Santos. O aluno com TDAH: Problematização diagnóstica e inclusão da escola. (Trabalho de Conclusão de Curso). Brasília: UnB. 2019, 41 p. Faculdade de Educação Brasília-DF. Disponível em: https://bdm.unb.br/handle/10483/22099. Acesso: 15 maio. 2021.

LOPES, Maria da Luz Curado. Inclusão, ensino e aprendizagem do aluno com TDAH. (Monografia). Universidade de Brasília - UnB. Instituto de Psicologia - IP. Brasília, 2011. Disponível em: https://bdm.unb.br/ handle/10483/2187. Acesso: 23 jun. 2021.

LOPES, Regina Maria Fernandes; NASCIMENTO, Roberta Fernandes Lopes do; BANDEIRA, Denise Ruschel. Avaliação do transtorno de déficit de atenção/ hiperatividade em adultos (TDAH): uma revisão de literatura. Avaliação Psicológica, n. 4 v. 1, 2005, p. 65-74. Disponível em: https:// dialnet.unirioja.es/descarga/articulo/6674820.pdf. Acesso: 13 jul. 2021.

MATTOS, Paulo et al. Painel brasileiro de especialistas sobre diagnóstico do transtorno de déficit de atenção/hiperatividade (TDAH) em adultos. Rev Psiquiatr RS jan/abr 2006; n. 28, v. 1, p. 50-60. Disponível em: https:// www.scielo.br/scielo.php?pid=S0101-81082006000100007\&script $=$ sci arttext\&tlng=pt. Acesso: 13 jul. 2021.

SCHMITZ, Marcelo et al. TDAH: remissão na adolescência e preditores de persistência em adultos. J. Bras. Psiquiatr. n. 56, supl 1, p. 25-29, 2007. Disponível em: https://www.scielo.br/scielo.php?pid=S004720852007000500006\&script=sci_arttext\&tlng=pt. Acesso: 02 set. 2021.

SILVA, Ana Beatriz Barbosa. Mentes inquietas TDAH: desatenção, hiperatividade e impulsividade. [livro eletrônico] - Rio de Janeiro: Objetiva, 2010. Disponível em: https://books.google.com.br/books?hl=pt-B $\mathrm{R} \& 1 \mathrm{r}=\& \mathrm{id}=\mathrm{NTtGBQAAQBAJ} \&$ oi $=\mathrm{fnd} \& \mathrm{pg}=\mathrm{PT} 7 \& \mathrm{dq}=\mathrm{SILVA},+\mathrm{Ana}+\mathrm{Beatr}$ iz+Barbosa.+Mentes+inquietas+TDAH:+desaten $\% \mathrm{C} 3 \% \mathrm{~A} 7 \% \mathrm{C} 3 \% \mathrm{~A} 3 \mathrm{o}$, +hip eratividade $+\mathrm{e}+$ impulsividade. $+\% 5$ Blivro+eletr $\% \mathrm{C} 3 \% \mathrm{~B} 4$ nico $\% 5 \mathrm{D}+-+\mathrm{Rio}+\mathrm{d}$ e+Janeiro:+Objetiva, +2010 .\&ots=-7z0Jw1Do3\&sig=ZEprWUqInoecjDs9v cpihTho7zo. Acesso: 02 set. 2021. 